\title{
Long-term neurological outcome of a cohort of 80 patients with classical organic acidurias
}

Mathilde Nizon ${ }^{1}$, Chris Ottolenghi ${ }^{1,2}$, Vassili Valayannopoulos', Jean-Baptiste Arnoux', Valérie Barbier ${ }^{1}$, Florence Habarou ${ }^{1,2}$, Isabelle Desguerre ${ }^{3}$, Nathalie Boddaert ${ }^{4}$, Jean-Paul Bonnefont ${ }^{5}$, Cécile Acquaviva ${ }^{6}$, Jean-François Benoist ${ }^{7}$, Daniel Rabier ${ }^{1}$, Guy Touati ${ }^{1}$ and Pascale de Lonlay ${ }^{1 *}$

\begin{abstract}
Background: Classical organic acidurias including methylmalonic aciduria (MMA), propionic aciduria (PA) and isovaleric aciduria (IVA) are severe inborn errors of the catabolism of branched-chain amino acids and odd-numbered chain fatty acids, presenting with severe complications.

Methods: This study investigated the long-term outcome of 80 patients with classical organic aciduria (38 with MMA, 24 with PA and 18 with IVA) by integrating clinical, radiological, biochemical and genetic data.

Results: Patients were followed-up for a mean of 14 years [age 3.3-46.3 years]. PA included a greater number of patients with abnormal neurological examination (37\% in PA, 24\% in MMA and 0\% in IVA), lower psychometric scores (abnormal evaluation at age 3 years in 61\% of patients with PA versus 26\% in MMA and 18\% in IVA) and more frequent basal ganglia lesions (56\% of patients versus $36 \%$ in MMA and $17 \%$ in IVA). All patients with IVA presented a normal neurological examination and only $1 / 3$ presented cognitive troubles. Prognosis for MMA was intermediate. Biochemical metabolite analysis excluding acute decompensations revealed significant progressive increases of glycine, alanine and glutamine particularly in PA and possibly in MMA but no correlation with neurological outcome. A significant increase of plasma methylmalonic acid was found in MMA patients with intellectual deficiency (mean level of $199 \mu \mathrm{mol} / \mathrm{L}$ versus $70 \mu \mathrm{mol} / \mathrm{L}, \mathrm{p}<0.05$ ), with an estimated significant probability of severe outcome for average levels between birth and age 6 years above $167 \mu \mathrm{mol} / \mathrm{L}$. Urinary 3-hydroxypropionate (3-HP) levels were significantly higher in PA patients with intellectual deficiency (mean level of $68.9 \mu \mathrm{mol} / \mathrm{mmol}$ of creatinine versus $34.6 \mu \mathrm{mol} / \mathrm{mmol}$ of creatinine, $p<0.01$ ), with an estimated significant probability of severe outcome for average levels between birth and age 6 years above $55 \mu \mathrm{mol} / \mathrm{mmol}$. As for molecular analysis, prognosis of MMA patients with mutations involving the MMAA gene was better compared to patients with mutations involving the MUT gene.
\end{abstract}

Conclusion: Propionic aciduria had the most severe neurological prognosis. Our radiological and biochemical data are consistent with a mitochondrial toxicity mechanism. Follow-up plasma MMA and urinary 3-HP levels may have prognostic significance calling for greater efforts to optimize long-term management in these patients.

Keywords: Organic aciduria, Propionic aciduria, Methylmalonic aciduria, Isovaleric aciduria, Neurological evolution, Long-term prognosis, Mitochondrial dysfunction

\footnotetext{
* Correspondence: pascale.delonlay@nck.aphp.fr

${ }^{1}$ Centre de Référence des Maladies Héréditaires du Métabolisme, Hôpital Necker-Enfants Malades, APHP, Université Paris Descartes, Institut Imagine, Paris, France

Full list of author information is available at the end of the article
} 


\section{Background}

The classical organic acidurias are branched-chain amino acids disorders involving intermediate metabolism. Depending on the enzymatic block, three major organic acidurias have long been distinguished: isovaleric aciduria (IVA) designates an isovaleryl-coA deshydrogenase defect on the leucine degradation pathway secondary to mutation in the IVD gene, propionic aciduria (PA) results from a defect in propionyl-coA carboxylase encoded by the $P C C A$ or $P C C B$ genes, and methylmalonic aciduria (MMA) is caused by mutations in the $M U T$ gene, encoding the methylmalonyl-CoA mutase (MCM), or more rarely in genes encoding the coenzyme adenosylcobalamin of MCM. $\mathrm{MCM}$ converts methylmalonyl-CoA into succinyl-CoA, an intermediate of the tricarboxylic acid cycle, the major source of NADH used by the mitochondrial respiratory chain. Two of these diseases, PA and MMA, affect the pathway common to valine, isoleucine, methionine and threonine metabolism.

Organic acidurias (OA) usually present as acute metabolic distress at birth with coma associated with dehydration when the enzymatic deficiency is complete, or later in life with recurrent ketoacidotic episodes, psychomotor retardation and chronic vomiting when the deficiency is partial $[1,2]$. As propionate is produced by the catabolism of branched-chain amino acids, fatty acids with a carbon odd-chain and the intestinal flora, the treatment is based on a strict low-protein diet associated with sufficient caloric intake, carnitine and antibiotics. However, despite therapeutic improvements over the last 20 years, overall outcome of patients with OA remains unsatisfying. Reports are increasing of long-term complications, such as neurological disorders by degeneration of the basal ganglia, progressive renal failure in MMA, cardiomyopathy in PA and acute pancreatitis in all [3-5].

Specific changes in the levels of urinary and plasma metabolites are the hallmark of the classical forms of the diseases including ketoacidosis, hyperlactatemia, hyperamoniemia, cytopenia in variable proportions. In urine, several organic acids are quite specific for diagnosis, particularly 3-hydroxypropionate and methylcitrate in PA, methylmalonate in MMA and isovalerylglycine in IVA. Enzymatic and genetic analyses confirm the diagnosis.

The pathophysiology of these disorders is not clearly understood and the proposed mechanisms are complex. Metabolite accumulation upstream of the enzymatic block triggers a systemic endogenous intoxication. Furthermore, the metabolic pathway involved in classical organic acidurias contributes to acetyl-coA and succinyl-coA formation, which are required for the tricarboxylic acid cycle. Thus, there is an energetic deficit by mitochondrial dysfunction secondary to substrate insufficiency and specific toxic metabolite accumulation including 3-hydroxypropionate [1].
The long-term neurological prognosis of these disorders depends on the severity of the disease, the delay of diagnosis and probably specific biochemical and genetic parameters. In particular, several studies attempting the delay to describe the neurological evolution of organic acidurias were hampered by the wide phenotypic variability even in a homogenous genetic population [6]. In order to better delineate the long-term neurological outcome of patients with organic acidurias, we analyzed clinical, radiological, biochemical and genetic parameters of a large cohort of patients.

\section{Methods}

\section{Patient cohort}

Inclusion criteria was a positive biochemical diagnosis of propionic, methylmalonic or isovaleric aciduria in a cohort of patients followed up at Necker-Enfants Malades hospital from 1991 to 2012 and managed with similar chemical and diet therapy. Exclusion criteria were an age less than 3 years old at the time of study, death before age 3 years or insufficient clinical data.

Neonatal onset refers to patients diagnosed within the first month. "Late onset" patients were all those diagnosed thereafter.

\section{Neurological evaluation}

Neurological evaluation was based on retrospective clinical data available in medical reports and was completed by radiological data (brain scanner and magnetic resonance imaging).

\section{Psychometric evaluation}

Each patient's psychometric evaluation was supported by school data and psychometric tests depending on the age of the patient and the evaluation year (Brunet Lezine from 0 to $2 \frac{1}{2}$ years old, Brunet Lezine with complementary test from 2 to 6 years old, WPPSI-III from 3 to 7 years old, NEMI from 4 to 12 years old and WISC-R and WISC-IV from 6 to 17 years old). Heterogeneity of the tests made it impossible to carry out a precise comparative study, thus we distinguished four distinct clinical groups based on developmental or intellectual quotient and autonomy level: group 0 with IQ $>85$ or DQ $>90$ in normal school, group 1 with $\mathrm{IQ}=71-85$ or $\mathrm{DQ}=76-90$ in normal school with specialized assistance for children in difficulties, group 2 with $\mathrm{IQ}=51-70$ or $\mathrm{DQ}=56-75$ in specialized classroom within normal school presenting mild intellectual deficiency and group 3 with $\mathrm{IQ} \leq 50$ or $\mathrm{DQ} \leq 55$ in specialized establishment presenting moderate to severe intellectual deficiency.

Neurological evaluation was performed at 1, 3, 6, 11 and 18 years old leading to the definition of four groups in term of cognitive evolution: group A with autonomy and good evolution (group 0 or 1 maintained from age 1 to 18 years), group $\mathrm{B}$ with improvement of psychometric 
evaluation over time (transition from initial classification into group 2-3 to classification into group $0-1$ ), group $C$ with worsening of psychometric evaluation (from group 0-1 to group 2-3) and group D with poor outcome from the outset and absence of autonomy.

\section{Acute decompensations}

Five different clinical conditions of variable severity were defined and reported on different periods from 0 to 3 , from 3 to 6 , from 6 to 11 and from 11 to 18 years old: 1 ) conditions at risk of acute decompensation needing an emergency diet at home; 2) conditions at risk of acute decompensation needing hospitalization for emergency diet by naso-gastric tube or intravenously without biological findings; 3) clinical and biological decompensation including $\mathrm{pH}<7.30$, bicarbonatemia $<18 \mathrm{mmol} / \mathrm{L}$, ammoniemia $>70 \mu \mathrm{mol} / \mathrm{L}$ or neurological trouble, 4) severe acute decompensation with intensive care and 5) severe acute decompensation with haemofiltration.

\section{Biochemical analysis}

Standard biochemical blood analysis included ammoniemia, lactate, amino acids (with particular emphasis on glycine, alanine, glutamine) and propionylcarnitine during basal state and acute decompensation, as well as $\mathrm{pH}$, bicarbonatemia during acute decompensation. Specific metabolites included urinary 3-hydroxypropionate, propionylglycine, tiglyglycine and methylcitrate for PA, plasma and urinary methylmalonate for MMA and urinary isovalerylglycine for IVA. Amino acids were assayed by ninhydrin colorimetry (Jeol Aminotac Analyzers) and organic acids by gas chromatography-mass spectrometry (Varian Saturn-2000).

\section{Genetic analysis}

Organic acidurias are recessive autosomal genetic diseases. Mutations in the PCCA (Propionyl CoA Carboxylase, alpha subunit, GenBank:NG_008768) and PCCB (Propionyl CoA Carboxylase, beta subunit, GenBank:NG_008939) genes for PA, MUT (Methylmalonyl CaA mutase, GenBank:NG_007100), MMAA (Methylmalonic Acidemia, cblA type Gene, GenBank:NG_007536) and $M M A B$ (Methylmalonic Acidemia, cblB type Gene, GenBank: NG_007096) genes for MMA and IVD (Isovaleryl Coa dehydrogenase, GenBank:NG_011986) for IVA were identified after PCR amplification of patient's DNA and dideoxynucleotide sequencing using the BigDye terminator cycle sequencing kit (Applied Biosystems, Foster City, CA, USA) and analysis with ABI Prism 3100 DNA sequencer.

\section{Statistical analysis}

The statistical analysis consisted in comparison of means by the two-tailed Student test with significance accepted for $\mathrm{p}<0.05$. Comparisons involving more than two groups were performed with one-way ANOVA followed by the Tukey's post-hoc test or two-way ANOVA. Logistic regression was performed with the bayesglm module in $\mathrm{R}$.

\section{Results}

Demographic analysis of the cohort of patients

The cohort included 80 patients from 74 families, 46 boys and 34 girls, born after 1990 and being alive after age 3 years, including 24 PA, 38 MMA and 18 IVA. Clinical follow-up of the cohort is shown in Figure 1. Mean age was 14 years and the median was 12.3 years [3.3-46.3 years old]. $58 \%$ of patients have been diagnosed during the first month (median current age, 11.4 years old). $42 \%$ of patients had a presentation from 1 month to 18 years old (median current age, 13.2 years old). Metabolic dysfunction led to liver transplantation for one PA patient and kidney transplantation for two MMA patients. Concerning MMA patients, only two sibling patients were sensitive to B12 supplementation. Thus, no correlation could be established with this series. Finally, one patient with PA died at 19 years old of an accidental fall, one with MMA died at 17 years old of a gastrointestinal bleeding after hernia surgery, and two patients with MMA died following an acute metabolic episode at 11 and 7 years old respectively.

\section{Clinical neurological and psychometric evaluation}

Clinical neurological examination was considered normal in $63 \%$ of PA (median age of 13 years old [3.8-46.3 years old]), $76 \%$ of MMA (median age of 10.9 years old [3.3-33.4 years old]) and $100 \%$ of IVA (median age of 11.9 years old [3.4-25.3 years old]) $(\mathrm{p}<0.01$ between IVA and MMA or PA, see Figure 2). Among the 18 patients with abnormal neurological examination, $33 \%$ had a pyramidal syndrome, $44 \%$ had an extrapyramidal syndrome, 33\% a cerebellar syndrome and $28 \%$ of the patients required assistance to walk ( $44 \%$ for PA, 11\% for MMA and none for IVA).

Moreover, seven patients were found with psychiatric disorders. Among PA, two teenagers presented with a psychotic disorder with hallucinations and disorganized behavior that was triggered by a moderate metabolic decompensation and was controlled by antipsychotic treatment within a few months. Such therapy may need to be adapted in case of cardiomyopathy or long QT syndrome. A third PA patient had a depressive and sleep disorder and one had an anxiety disorder. Among MMA, two patients had behavioral disorder and one patient had a personality disorder with anxiety. Most of these patients were treated by psychotherapy and by conventional drugs. Among PA, one patient followed up to 46 years old had memory trouble with ictus amnesic episodes. 6 to $21 \%$ of patients had attention trouble and 8 to $33 \%$ of patients had fine motor trouble disturbing handwriting and requiring rehabilitation. 


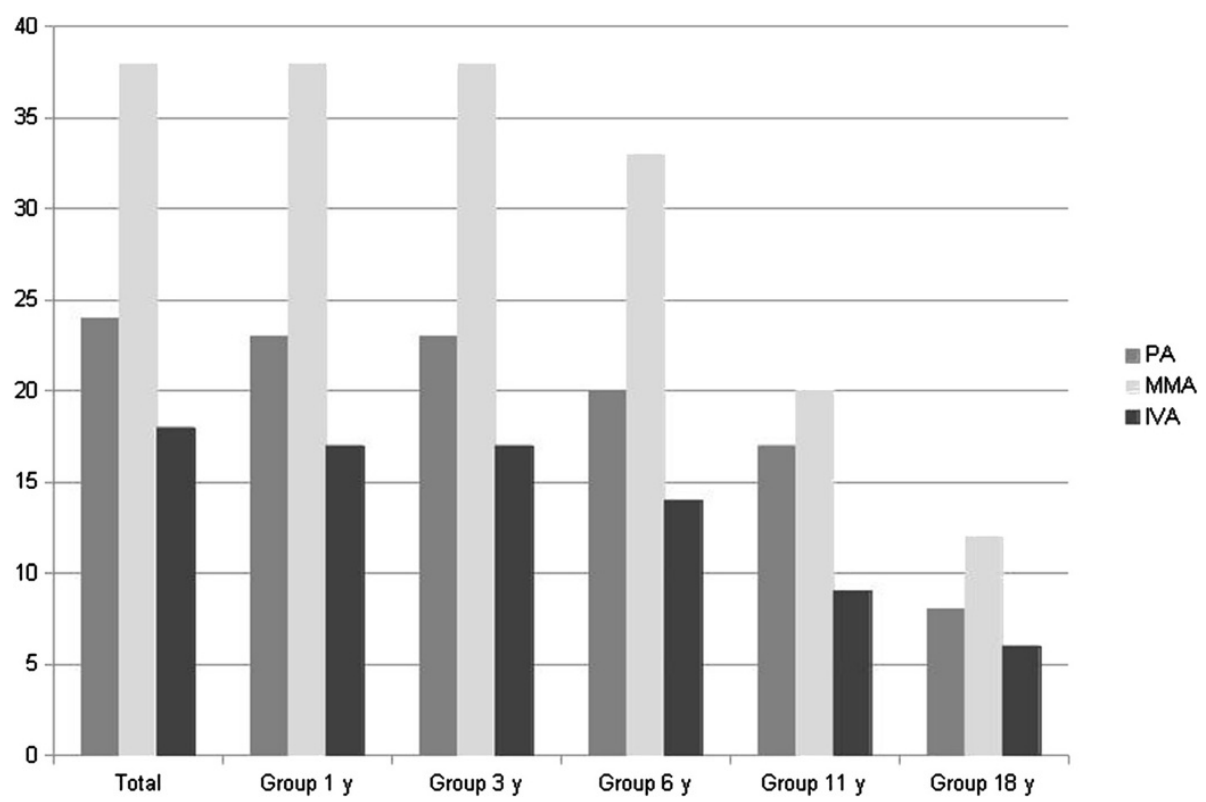

Figure 1 Number of patients with available clinical data according to age. PA: propionic aciduria, MMA: methylmalonic aciduria, IVA: isovaleric aciduria; $y$ : years.

Psychometric evaluation at different times highlighted a specific pattern attendant on the type of organic aciduria (Figures 3 and 4). The score was considered as normal (groups 0 and 1) in $39 \%$ and $47 \%$ of patients with PA at ages 3 and 11 years respectively, in $74 \%$ and $50 \%$ of patients with MMA at ages 3 and 11 years respectively, and $82 \%$ and $67 \%$ of patients with IVA at ages 3 and 11 years respectively. Interestingly, at age 11 years, group 0 included only a few patients: 4/20 for MMA, 1/9 for IVA and 0/17 for PA. By contrast, at this age, 29\% of PA (12/17), 25\% of MMA (15/20) and $11 \%$ of IVA (1/9) had deficiency in expression and comprehension skills.

PA patients were neurologically more severely affected than MMA and IVA at 3 years of age, with $61 \%$ of PA patients in group 2 or 3 compared to $26 \%$ for MMA and $18 \%$ for IVA ( $<0.01$ between PA and MMA or IVA, ANOVA test). The outcome was good for only $12 \%$ of patients with PA comparing to 50\% MMA patients and $67 \%$ IVA patients (group A). Of note, the data indicated

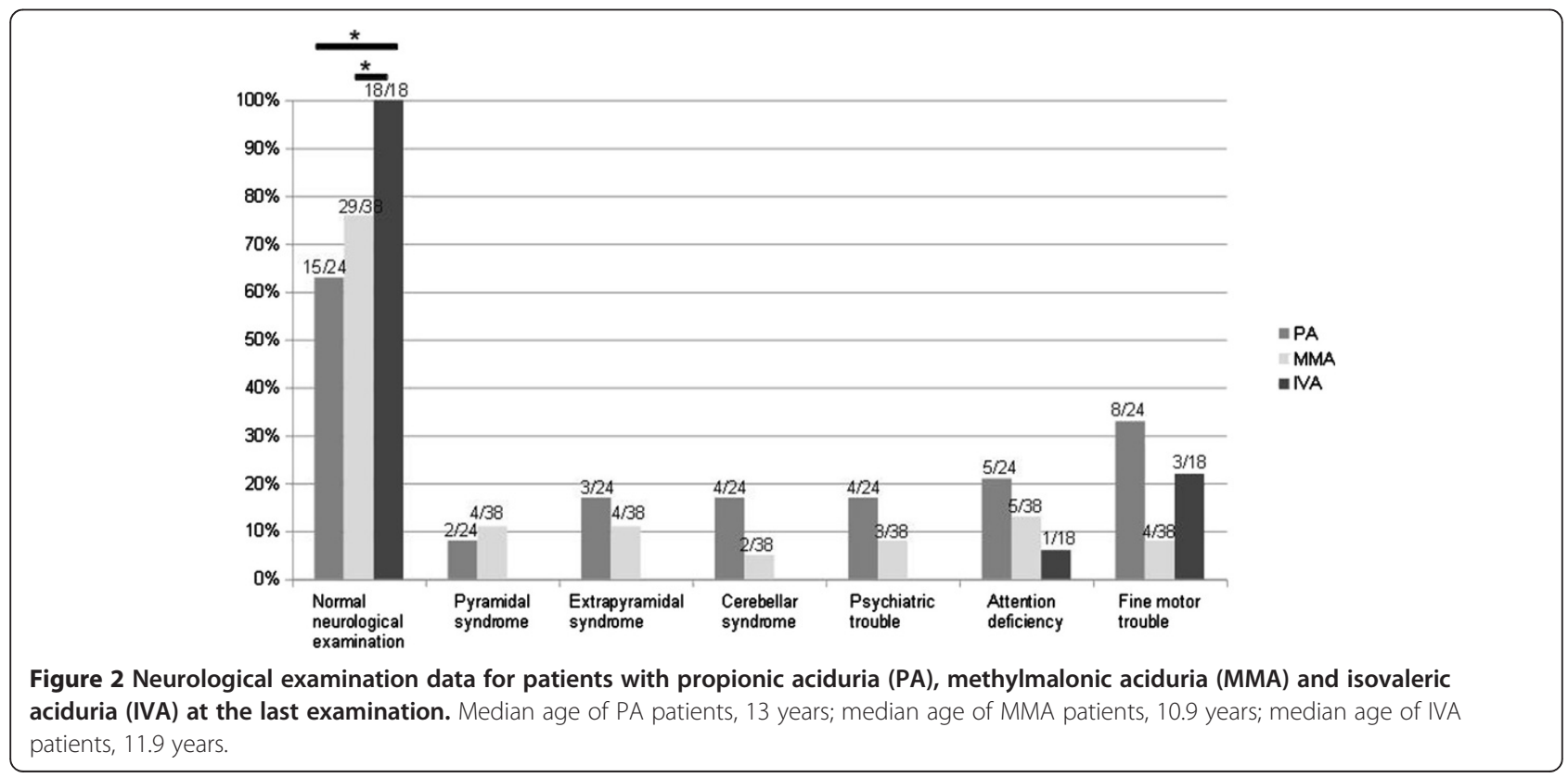




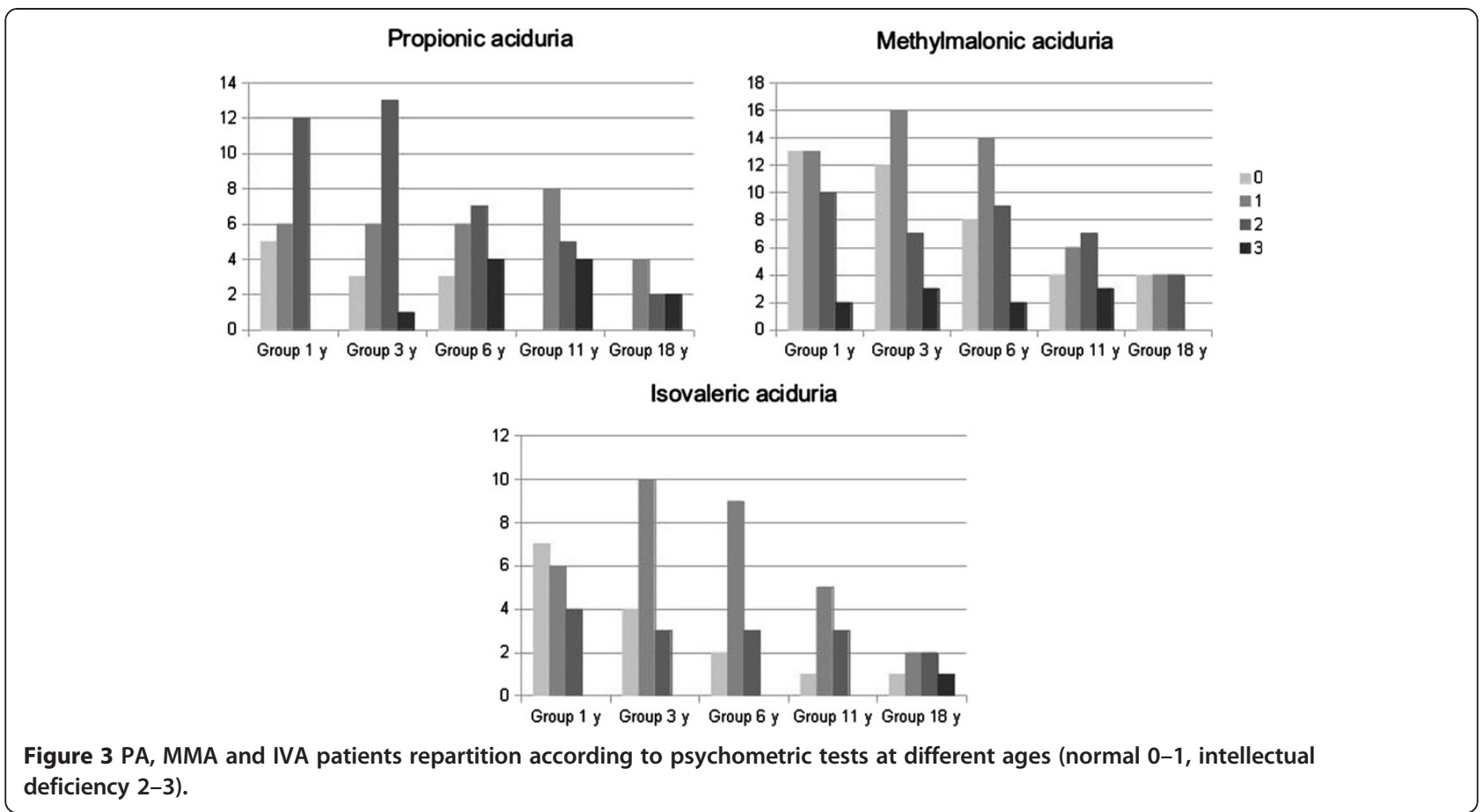

psychometric improvement in 35\% of PA patients from group $B$ and not in other organic aciduria. Then, at 11 years old, groups $\mathrm{A} / \mathrm{B}$ and $\mathrm{C} / \mathrm{D}$ proportion was about the same among the three organic acidurias with a better prognosis for IVA. Furthermore, cognitive regression concerned 20 to $29 \%$ patients for all three organic acidurias (group $\mathrm{C}$ at 11).

\section{Correlation between age of onset and neurological examination}

Neurological examination was normal for $87 \%$ of patients with neonatal diagnosis and for $66 \%$ of patients diagnosed later $(\mathrm{p}<0.05$, see Figure 5$)$. Both groups had pyramidal syndrome, cerebellar syndrome, psychiatric troubles, attention deficiency or fine motor troubles in insignificant different proportions. Only late onset diagnosis patients

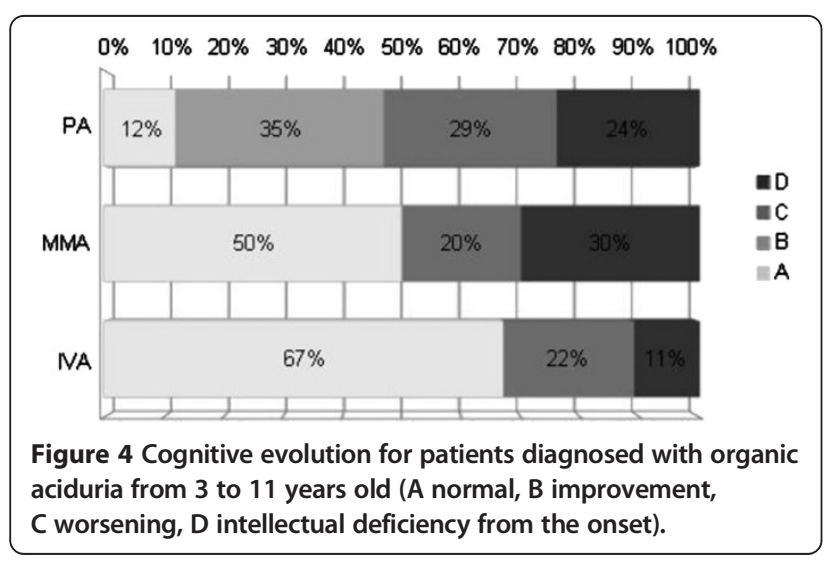

had an extrapyramidal syndrome, with frequent difficulty to walk: 3/4 PA and 1/4 MMA required assistance to walk.

\section{Correlation between date of onset and neurological data}

Between 1991 and 2000, the neonatal resuscitation was less effective than after year 2000 and nocturnal nutrition by nasogastric tube was not systematic at that time contrary to current recommendations. Therefore, we reanalyzed data in two subgroups, depending on whether diagnosis was done before or after 2000. A fraction of $48 \%$ of the patients was diagnosed before 2000 with similar proportions among the different organic acidurias. Neurological examination was normal for $68 \%$ of patients who were diagnosed between 1991 and 2000 (50\% of PA, 68\% of MMA, $100 \%$ of IVA) and for $86 \%$ of patients diagnosed after year 2000 (75\% of PA, $84 \%$ of MMA, $100 \%$ of IVA, p $=0.07$, not significant). Psychometric tests showed no significant difference between the two groups: IQ was normal at 6 years old for $50 \%$ of patients diagnosed between 1991 and 2000 and $71 \%$ of patients diagnosed after 2000 ( $p=0.08$, not significant). Therefore, there was a non-significant trend toward better neurological scores (neurological examination and psychometric tests) for patients diagnosed after 2000, consistent with improved management.

\section{Brain imaging evaluation}

Imaging was available for 50/80 patients including 16/24 PA, 28/38 MMA and 6/18 IVA. Brain MRI was normal for $19 \%$ of PA, $46 \%$ of MMA and $33 \%$ of IVA when 


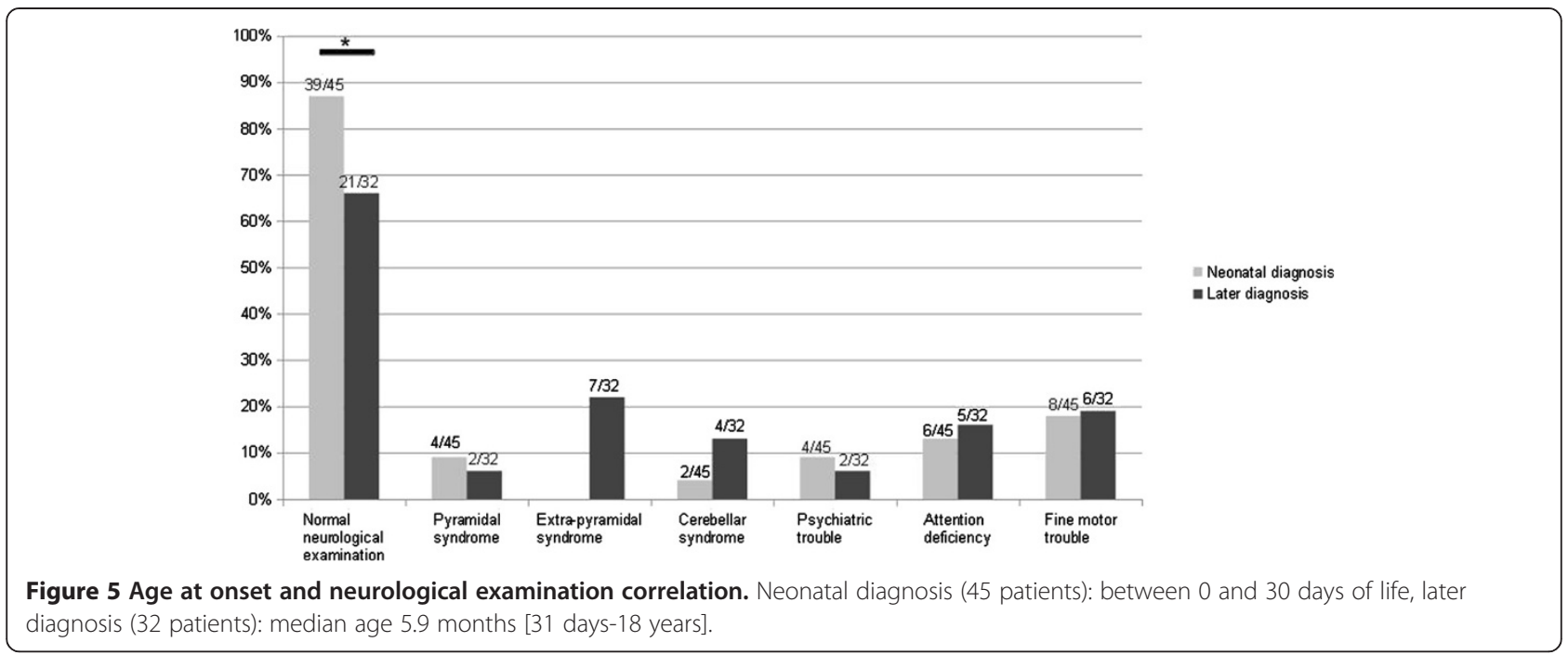

available (see Figure 6). Abnormalities included basal ganglia lesions for $56 \%$ of PA, $36 \%$ of MMA and $17 \%$ of IVA, notably involving the striatum and the globus pallidum, white matter anomalies for 38 to $50 \%$ and cerebellar anomalies for three MMA and one IVA. For five patients, normalization of basal ganglia lesions and white matter anomalies were observed at a distance from the acute episode. Furthermore, two patients PA and three patients MMA had increased lactate concentration in basal ganglia. Magnetic resonance spectroscopy was not performed for the other patients.

Brain imaging and neurological examination correlation Among 62 patients with normal neurological examination, 30 patients had available brain imaging: the pictures were normal for $47 \%$ of patients, and showed white matter anomalies for $40 \%$, basal ganglia anomalies for $17 \%$ and cerebellar abnormalities for $10 \%$.
Among the six patients with pyramidal syndrome, two had normal brain MRI, three had basal ganglia anomalies, two had white matter anomalies and one cerebellar atrophy. Among the seven patients with extra-pyramidal syndrome, two had a normal brain MRI, four had basal ganglia anomalies and two had white matter anomalies. Of the four patients with cerebellar syndrome, two had a normal MRI and two had white matter anomalies. There was no correlation between long-term psychometric test evolution and brain anomalies.

\section{Acute decompensations}

Excluding initial decompensation, PA patients underwent an average of 1.8 acute decompensations from birth to age three years [0-8] whereas IVA patients had only 0.5 [0-2] and MMA patients 1.2 [0-7] (see Figure 7); the difference between PA and IVA was significant $(\mathrm{p}<0.05$; we did not count the initial decompensation). For the three

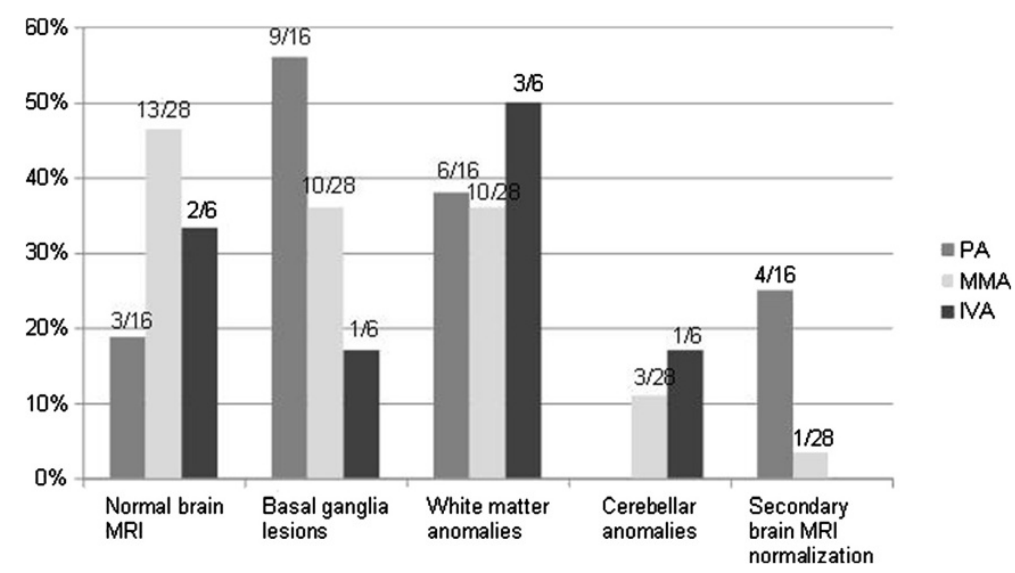

Figure 6 Brain MRI data for 16 PA patients, 28 MMA patients and 6 IVA patients; median 13.1 years. Secondary brain MRI normalization performed in 5 patients with a follow-up of 5 years. 


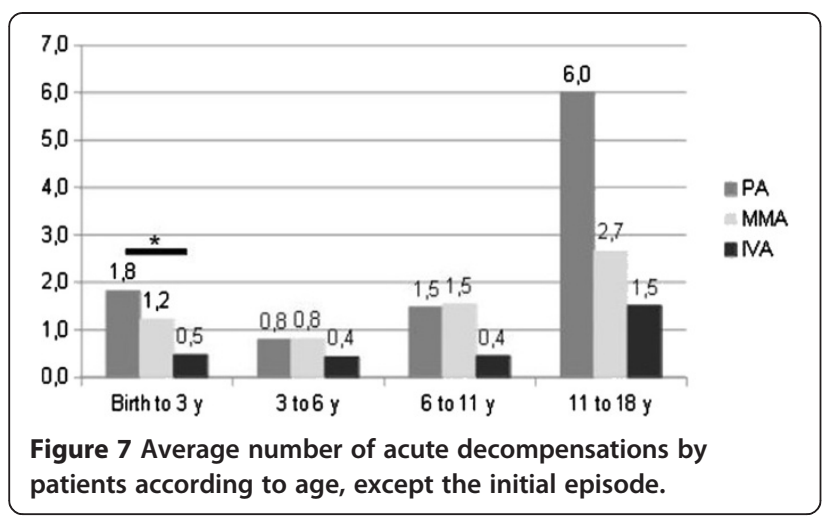

organic acidurias, we found two frequency peaks of decompensations, from birth to three years old and from eleven to eighteen years old. Furthermore, the number of patients requiring haemofiltration for one of the acute decompensations including the initial episode was $6 / 24$ for PA (two patients required two and three haemofiltrations respectively), 7/38 MMA and 1/8 IVA. Except for one of the seven MMA patients, haemofiltration was performed only at the first decompensation.

\section{Biochemical profile outside of acute decompensations}

Plasma amino acid levels differed significantly with disease status and age, showing a highly significant interaction for glycine $(\mathrm{p}=0.0001$; Table $1 \mathrm{~A})$. Indeed compared to MMA and IVA, the pattern of variation of glycine was strikingly specific to PA, showing a sharp increase at an early age and a high plateau at about 11 years (Additional file 1, Figure 8). In addition, the two other tested amino acids, glutamine and alanine, showed highly significant increases with age specifically in PA, whereas variations were essentially

Table 1 Plasma amino acid levels in relation to disease and age

\begin{tabular}{|c|c|c|c|}
\hline \multicolumn{4}{|c|}{ 1A. Joint effect of disease and age on plasma amino acid levels } \\
\hline & Disease & Age & $\begin{array}{l}\text { Age and disease } \\
\text { interaction }\end{array}$ \\
\hline Plasma alanine & $0.0010^{* *}$ & $0.0069^{* *}$ & $0.027^{*}$ \\
\hline Plasma glutamine & $0.0000^{* * *}$ & $0.0012^{* *}$ & $0.030^{*}$ \\
\hline Plasma glycine & $0.0000^{* * *}$ & $0.0000^{* * *}$ & $0.0001^{* * *}$ \\
\hline \multicolumn{4}{|c|}{ 1B. Effect of age on plasma amino acid levels for each disease } \\
\hline & IVA & MMA & PA \\
\hline Plasma alanine & 0.051 & 0.068 & $0.0041^{* *}$ \\
\hline Plasma glutamine & 0.17 & 0.39 & $0.0005^{* *}$ \\
\hline Plasma glycine & 0.22 & $0.011^{*}$ & $0.0000^{* * *}$ \\
\hline
\end{tabular}

A: Joint effect of disease status and age on plasma amino acid levels (two-way ANOVA, p-values). B: Effect of age on plasma amino acid levels for each disease (one-way ANOVA, p-values). To enhance readability, p-values are rounded following the first non-zero digit or at total four digits. Significance cutoffs for p-values: ${ }^{*}<0.05 ;{ }^{* *}<0.01 ;{ }^{* *}<0.001$. borderline in MMA, and undetectable or not progressive with age in IVA (Figure 8 and Table 1B). There was no correlation between plasma glycine, glutamine and alanine levels and the neurological, psychometric evolution and brain anomalies for any age group.

Organic acid levels average over the first six years was significantly associated with neurological prognosis for MMA and PA. Indeed, plasma methylmalonic acid was $70 \mu \mathrm{mol} / \mathrm{L}$ for MMA group A/B and $199 \mu \mathrm{mol} / \mathrm{L}$ for MMA group $C / D(p<0.05)$. By logistic regression, the odds ratio for severe outcome (groups C/D rather than A/B) was 4.3 (95\% confidence interval: 1.1-16.3) for every two-fold multiplicative increase of average plasma MMA levels over the first 6 years, and the risk of severe outcome was significant for values greater than or equal to $167 \mu \mathrm{mol} / \mathrm{L}$ (probability: $0.67 ; 95 \%$ confidence interval: 0.51-0.84).

As for urinary 3-hydroxypropionate, the average level over the first 6 years was $34.6 \mu \mathrm{mol} / \mathrm{mmol}$ of creatinine for PA group $\mathrm{A} / \mathrm{B}$ and $68.9 \mu \mathrm{mol} / \mathrm{mmol}$ of creatinine for PA group $\mathrm{C} / \mathrm{D}(\mathrm{p}<0.01)$. Odds ratio for severe outcome was 5.1 for every two-fold multiplicative increase of urinary 3-hydroxypropionic acid levels averaged over the first 6 years. The risk of severe outcome was significant for values greater than or equal to $55 \mu \mathrm{mol} / \mathrm{L}$ (probability: 0.64; 95\% confidence interval: 0.51-0.77)

Other metabolites were not correlated with clinical evolution, notably urinary isovalerylglycine for IVA (1556 $\mu \mathrm{mol} / \mathrm{mmol}$ of creatinine), plasma propionylcarnitine level for MMA $(45.4 \mu \mathrm{mol} / \mathrm{L})$, lactatemia $(2 \mathrm{mmol} / \mathrm{L})$ and ammoniemia $(54 \mu \mathrm{mol} / \mathrm{L})$ for PA, MMA and IVA.

\section{Genetic analysis}

The genotype was known for 49/80 patients (see Table 2). Two PA patients had mutation in PCCA (one stop, one missense and one splice mutations) and seven in $P C C B$ (five stop and five missense mutations), eight MMA patients had mutation in $M M A A$ (seven stop and one missense mutations), one in $M M A B$ (stop mutation) and 29 in $M U T$ (nine stop and sixteen missense mutations including two recurrent mutations p.Ala191Glu for 19\% and p.Asn219Tyr for 13\%) and five IVA patients had mutation in IVD (four missense mutations).

For PA patients, there was no correlation between genotype and neurological evolution, especially between patients with two stop mutations and those with two missense mutations. The number of patients was not sufficient for any in-depth investigation.

For MMA patients, cognitive prognosis and neurological evolution were significantly better at age 6 or 11 years, for $M M A A$ mutated patients compared to patients with mutation in $M U T$. Indeed, 7/8 MMAA mutated patients and $9 / 26 M U T$ mutated patients were classified in the group A ( $\mathrm{p}<0.05$, Fisher's exact test) while $1 / 8 M M A A$ 


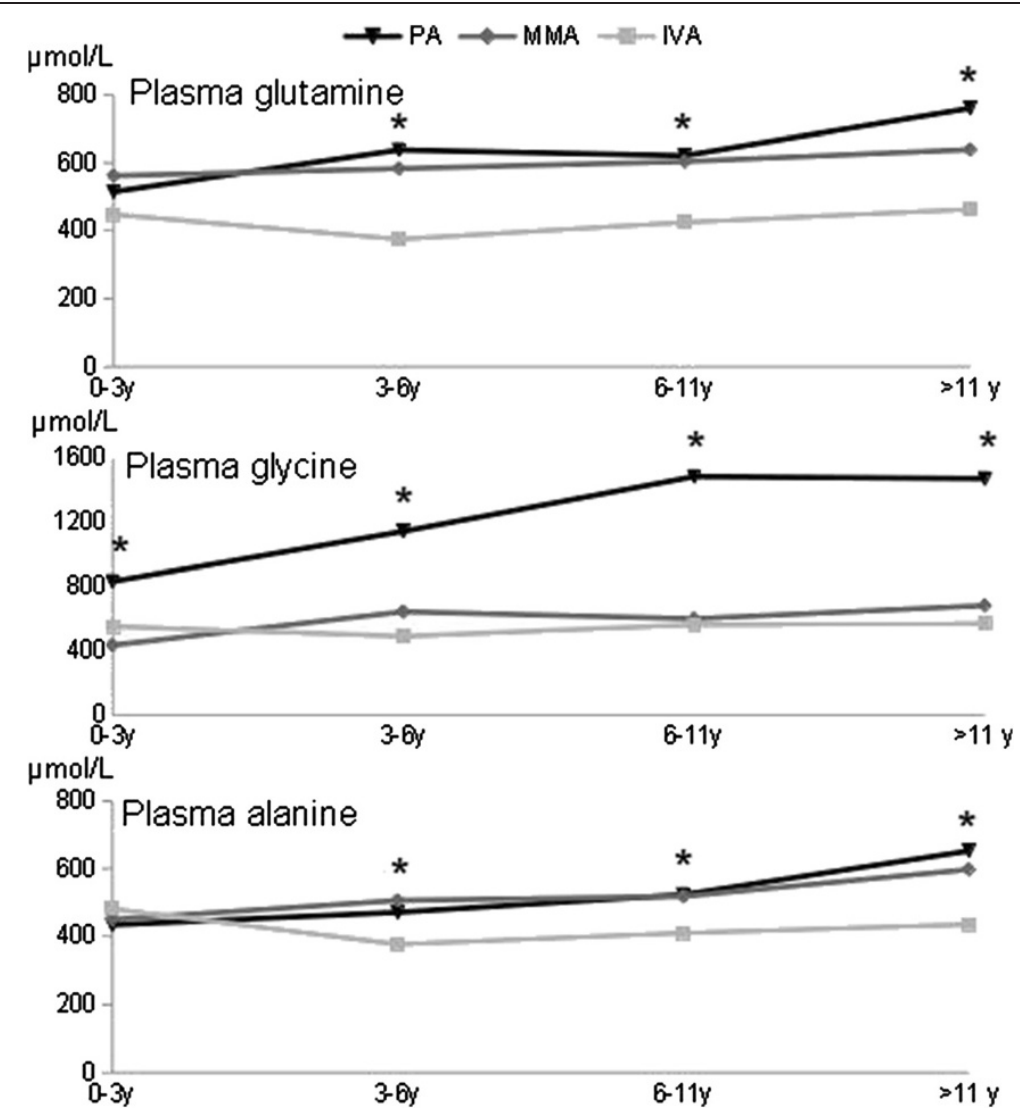

Figure 8 Evolution of the amino acid levels according to age by each disease $(*$ : $p$-values $:<0.01)$.

mutated patients and 7/26 MUT mutated patients had abnormal neurological examination. Furthermore, cognitive evolution at 6 or 11 years old seemed to be better for patients with mutation in $M U T$ with two missense mutations (7/12 in group A) compared to those with two stop mutations (four patients in group $\mathrm{C}$ or $\mathrm{D}$ ). However there was quite heterogenous neurological prognosis for patients with the same mutation.

We found no genotype phenotype correlation in IVA patients.

\section{Discussion}

This study investigates the long-term neurological prognosis of 80 patients presenting with classical organic acidurias, i.e. PA, MMA and IVA that involve the branched-chain amino acids metabolism. The results reinforce the notion that PA patients have the most severe neurological prognosis with $37 \%$ of patients with abnormal neurological examination, 61\% with abnormal cognitive test at age three years and $56 \%$ with basal ganglia lesions. PA patients also presented more frequent acute decompensations from zero to three years old (1.8 on average). Four patients had psychiatric troubles including delirious syndrome during an acute decompensation. However, long-term cognitive evaluation was similar to MMA because of an improvement of psychometric tests with $47 \%$ of PA patients achieving normal evaluation at 11, which was not paralleled by MMA patients. Recently, Grünert et al. [5] reported a cohort of 55 PA patients with intellectual deficiency in $75 \%$ of patients (median IQ at 55), hypotonia in $51 \%$ of patients and ataxia in $9 \%$ of patients. Brain MRI revealed basal ganglia lesions in $5 / 26$ patients [5]. We note that our data correspond to a psychometric evaluation performed at age 11 years. Most patients reported by Grünert et al. were younger (median age: 5.2 years [5]), which may explain why they did not (yet!) show the cognitive improvement that we have observed on average. Nevertheless in some of our patients, evolution rather involved a progressive neurological worsening.

IVA had the best neurological prognosis with normal neurological examination in all patients, though white matter anomalies were noted in 3 patients of 6 available MRI. However, fine motor trouble was detected in $22 \%$ of patients, as well as some degree of intellectual deficiency in one third of IVA patients. Our data complement a study realized on 16 IVA patients including $44 \%$ with coordination trouble or moderate motor trouble 
Table 2 List of mutations (nucleotidic or amino acid change depending on the patients) for patients diagnosed with PA, MMA and IVA and neurological evaluation

\begin{tabular}{|c|c|c|c|c|c|c|}
\hline Patient & Gene & Allele 1 & Allele 2 & Cognitive evolution at $11 \mathrm{y}$ & $\begin{array}{l}\text { Neurological examination } \\
\text { anomalies }\end{array}$ & $\begin{array}{l}\text { Brain MRI } \\
\text { anomalies }\end{array}$ \\
\hline 1 & PCCA & c. $1284+4 \mathrm{~A}>\mathrm{T}$ & p.Arg430X & A & no & \\
\hline 2 & PCCA & p.Ala138Thr & p.Ala138Thr & B & yes & yes \\
\hline 3 & $P C C B$ & p.Gln147GInfs X2 & c.1218_1231del14ins12 & $\mathrm{D}$ & no & no \\
\hline 4 & $P C C B$ & p.Glu331X & p.Glu331X & C & no & yes \\
\hline 5 & $P C C B$ & p.Glu331X & p.Glu331X & $\mathrm{D}$ & yes & yes \\
\hline 6 & $P C C B$ & p.Arg514X & p.Arg529Argfs X44 & & yes & \\
\hline 7 & $P C C B$ & p.Arg165Trp & $?$ & $\mathrm{~B}$ & no & \\
\hline 8 & $P C C B$ & p.Phe180Ser & p.Phe180Ser & & yes & yes \\
\hline 9 & $P C C B$ & p.His258Arg & p.Arg376Cys & B & no & no \\
\hline $10 a$ & MMAA & del exon2-3-4 & del exon2-3-4 & A & no & \\
\hline $10 \mathrm{~b}$ & MMAA & del exon2-3-4 & del exon2-3-4 & D & yes & yes \\
\hline 11 & MMAA & p.Arg22X & p.Arg22X & $A(6 y)$ & no & yes \\
\hline 12 & MMAA & p.Thr221del & p.Thr221del & A & no & \\
\hline 13 & MMAA & p.Arg145X & $?$ & A & no & \\
\hline 14 & MMAA & p.Arg196X & p.Arg196X & $A(6 y)$ & no & \\
\hline 15 & MMAA & c. 592_595del & c.594_598del & A & no & no \\
\hline 16 & MMAA & p.Lys276Asn & p.Lys276Asn & A & no & \\
\hline 17 & $M M A B$ & p.Arg186Trp & p.Arg190Cys & $A(6 y)$ & no & \\
\hline 18 & MUT & c.360_361insT & c.360_361insT & C & no & yes \\
\hline 19 & MUT & c.693_694insA & c.693_694insA & $D(6 y)$ & no & yes \\
\hline 20 & MUT & p.GIn352X & p.GIn352X & & no & \\
\hline 21 & MUT & p.Arg474X & p.Arg511X & C & yes & yes \\
\hline 22 & MUT & c.1876_1888del & c.1876_1888del & $\mathrm{D}$ & yes & yes \\
\hline 23 & MUT & p.Arg228X & p.Arg369Cys & & yes & no \\
\hline 24 & MUT & p.Ser342X & p.Arg93His & $B(6 y)$ & no & \\
\hline 25 & MUT & p.Ser342X & p.Arg694Trp & C & no & no \\
\hline 26 & MUT & p.Ser342X & p.Ala191Glu & $A(6 y)$ & no & yes \\
\hline 27 & MUT & c.662delT & p.Gly158Val & & no & no \\
\hline 28 & MUT & p.Arg511X & p.Gly642Arg & A & no & no \\
\hline 29 & MUT & p.Arg511X & p.Arg603Thr & $D(6 y)$ & no & no \\
\hline 30 & MUT & p.Ala191Glu & p.Ala191Glu & $D(6 y)$ & no & yes \\
\hline 31 & MUT & p.Ala191Glu & p.Ala191Glu & $\mathrm{D}$ & no & \\
\hline 32 & MUT & p.Ala191Glu & p.Ala191Glu & $A(6 y)$ & no & yes \\
\hline $33 a$ & MUT & p.Ala191Glu & p.Gly623Arg & $A(6 y)$ & no & \\
\hline $33 b$ & MUT & p.Ala191Glu & p.Gly623Arg & A & yes & no \\
\hline 34 & MUT & p.Gly203Arg & p.Arg108Cys & & no & yes \\
\hline 35 & MUT & p.Gly203Arg & p.Met700Lys & A & no & no \\
\hline 36 & MUT & p.Gly215Ser & p.Gly215Ser & $A(6 y)$ & no & no \\
\hline 37 & MUT & p.Asn219Tyr & p.Asn219Tyr & A & no & no \\
\hline 38 & MUT & p.Asn219Tyr & p.Asn219Tyr & & yes & no \\
\hline 39 & MUT & p.Asn219Tyr & p.Gln383His & $\mathrm{D}$ & yes & yes \\
\hline 40 & MUT & p.Asn219Tyr & p.Val162Phe & $B(6 y)$ & no & \\
\hline
\end{tabular}




\begin{tabular}{|c|c|c|c|c|c|c|}
\hline 41 & MUT & p.Asn219Tyr & p.Ala191Glu & $\mathrm{D}$ & yes & yes \\
\hline 42 & MUT & p.Thr230lle & p.Thr230lle & A & no & yes \\
\hline $43 a$ & IVD & p.Arg284GIn & p.Arg284GIn & & no & \\
\hline $43 b$ & IVD & p.Arg284Gln & p.Arg284GIn & A & no & \\
\hline 44 & IVD & p.Gly289Arg & p.Gly289Arg & & no & yes \\
\hline 45 & IVD & p.Cys378Arg & p.Cys378Arg & A & no & \\
\hline 46 & IVD & p.Arg392His & p.Arg392His & $B$ & no & \\
\hline
\end{tabular}

and $16 \%$ with intellectual deficiency [2]. In literature, neurological prognosis was good for $85 \%$ of IVA patients diagnosed during neonatal period while only for $45 \%$ of the patients diagnosed later. Eventually, some acute decompensations occurred during teenage years.

MMA had an intermediate prognosis between PA and IVA with $24 \%$ of abnormal neurological examination, $54 \%$ of brain anomalies and $50 \%$ of intellectual deficiency at 11 years old.

Identification of prognostic factors would be useful to prevent neurological complications and to guide long-term follow up. Early diagnosed patients had better neurological prognosis as neurological examination was normal for $87 \%$ of patients diagnosed during neonatal period but only $66 \%$ of those diagnosed later $(\mathrm{p}<0.05)$, as previously described in several studies, arguing in favor of neonatal screening for organic acidurias [7-9]. Most forms being revealed at a few post-natal days of life, neonatal screening will be effective for later onset forms. In this cohort, all patients with an extra-pyramidal syndrome were diagnosed after the neonatal period. By contrast, the number of acute decompensations and their severity did not influence the prognosis. Similarly, there is no correlation between brain anomalies and abnormal neurological examination or intellectual deficiency. Likewise, liver transplants for PA and MMA and kidney or combined liver-kidney transplant for MMA improve the metabolic status. Benefits are shown in term of significant improvement of quality of life with increased protein tolerance, reduction of acute decompensations and hospitalizations. However transplantation does not cure the disease. Metabolic decompensations are less common but remain possible and long-term complications, in particular neurological impairment, are not prevented [10]. This suggests that additional prognostic factors are involved, notably the degree of metabolite balance in the basal state. Considering standard metabolites, there was no correlation between the biochemical profile excluding the acute decompensations and neurological outcome. However, we found significant progressive increases of the three plasma amino acids tested in PA and not in IVA, with an intermediate pattern in MMA (Table 1 and Figure 8).
Because the deviating plasma amino acid pattern progresses with age, it might result from unsatisfactory management during follow-up rather than at diagnosis. This may be relevant to the finding that intellectual ability in our PA cohort is better at 11 years than reported at an earlier age in another cohort (see above) [5]. Thus, greater efforts may be needed to optimize long-term management in these patients.

Considering patients with MMA, plasma methylmalonic acid levels were higher in patients with intellectual deficiency, with an "alert" value estimated at $167 \mu \mathrm{mol} / \mathrm{L}$. In the same way, urinary 3-hydroxypropionate level was higher in patients with intellectual deficiency, with an "alert" value of $55 \mu \mathrm{mol} / \mathrm{mmol}$ of creatinine. Finally, genotype analysis was not informative for prognosis. As for PA, there was no genotype phenotype correlation presumably because of the small number of patients. Previous studies reported that the phenotype was more severe among patients with two stop mutations or with missense mutations altering protein stability, especially those involving $P C C B[11,12]$. As for MMA, global neurological prognosis was better for patients with mutations in $M M A A$ gene compared to those mutated in $M U T$ gene even if most mutations in $M M A A$ were stop mutations. These results can be explained by the role of the protein coded by $M M A A$ in synthesis of adenosylcobalamin, the cofactor of methylmalonyl-CoA mutase, and its occasional sensitivity to B12 supplementation. Consistent data have already been presented in previous studies [13,14]. As for IVA, molecular data were available for only five patients and there was no obvious genotype phenotype correlation. A previous study on a cohort of patients presenting with the same homozygous missense mutations showed great phenotypic variability [6].

Finally, clinical and biochemical differences observed between the three organic acidurias highlighted some differences of pathophysiological relevance. The best prognosis was associated with IVA, possibly because of a great efficacy of glycine to detoxicate accumulated metabolites. Furthermore, leucine degradation leads to acetyl-CoA and not succinyl-CoA formation contrary to PA and MMA, 
making the energy defect possibly less pronounced in IVA. This is also suggested by the absence of basal ganglia lesions and the normal lactate peak on magnetic resonance spectroscopy. An in vitro study on rat cortex synapses showed that IVA metabolites do not modify tricarboxylic cycle and respiratory chain activity but inhibits $\mathrm{Na}^{+}, \mathrm{K}^{+}$-ATPase, an enzyme playing a role in brain development and neuronal stability [15]. Phenotypic variability observed in a homogenous population suggests that other environmental or genetic factors modify isovaleryl-CoA deshydrogenase activity or the efficiency of glycino-conjugation [6]. Other metabolites, such as isovalerylglutamate, methylfumarate, isovalerylserine and 3-hydroxycaproate have recently been detected and could play a role in pathophysiology [16]. By contrast, PA generates several toxic metabolites such as 3hydroxypropionate, methylcitrate, and propionyl-CoA. We have shown that urinary 3-hydroxypropionate level correlates with long term neurologic outcome consistent with previous hypotheses based on propionate toxicity on glial cell metabolism [17]. Propionyl-CoA and methylcitrate are responsible for mitochondrial dysfunction by enzyme inhibition and propionyl-CoA decreases acetyl-coA formation by CoA sequestration $[18,19]$. These features may account for the severity of PA deficiency that frequently leads to basal ganglia lesions, high lactate peak on magnetic resonance spectroscopy and significant increase of plasma alanine level. Furthermore, increased plasma glycine is more pronounced in PA patients thus also arguing for an important dysfunction of the glycine cleavage system in mitochondria. In MMA, methylmalonic acid levels also correlated with long-term neurological outcome. Other metabolites such as 3-hydroxypropionate, methylcitrate, tiglyglycine and propionylgycine were present in much smaller amounts compared to PA. The intermediate prognosis of MMA suggests that toxicity may mainly result from intermediate levels of PA metabolites. Indeed, it has been demonstrated that methylmalonate has no obvious toxicity on respiratory chain [20], yet secondary respiratory chain deficiency has been reported in PA and MMA patients in different tissues [21,22].

\section{Conclusion}

In conclusion, this study of 80 patients with organic acidurias brings additional knowledge on long-term neurological outcome and highlights the need to identify relevant environmental or genetic prognostic factors that may modulate severity of the disorders. We have identified prognostic "alert" values for the two more severe deficiencies, MMA and PA. Our clinical, radiological and biochemical data strengthen the hypothesis of a mechanism of direct mitochondrial toxicity for PA and to a smaller degree, for MMA.

\section{Additional file}

Additional file 1: Average plasma amino acid levels +/- standard deviation according to age by each disease. All available tests were included (at least one per year) except those obtained during acute decompensations. PA: propionic aciduria, MMA: methylmalonic aciduria, IVA, isovaleric aciduria.

\section{Competing interests}

The authors declare that they have no competing interests.

\section{Authors' contributions}

MN designed the study, performed it and wrote the manuscript; CO performed statistical and biochemical analysis and participated in the manuscript preparation; W, JBA and GT described patient phenotype; VB performed psychometric tests; FH and DR performed biochemical analysis; ID and NB described neurological phenotype; JPB, CA and JFB performed molecular analysis; PdL designed the study and participated in the manuscript preparation. All authors read and approved the final manuscript.

Acknowledgements

We thank the "Jérôme Lejeune" foundation, patients and their families.

\section{Author details}

${ }^{1}$ Centre de Référence des Maladies Héréditaires du Métabolisme, Hôpital Necker-Enfants Malades, APHP, Université Paris Descartes, Institut Imagine, Paris, France. ${ }^{2}$ Service de Biochimie métabolique, Hôpital Necker-Enfants Malades, U747, Inserm - Université Paris Descartes, Paris, France. ${ }^{3}$ Service de Neurologie, Hôpital Necker-Enfants Malades, APHP, Université Paris Descartes, Paris, France. ${ }^{4}$ Service de Radiologie Pédiatrique, Hôpital Necker-Enfants Malades, APHP, Université Paris Descartes, Institut Imagine, Paris, France. ${ }^{5}$ Service de Génétique Médicale, Hôpital Necker-Enfants Malades, APHP, Université Paris Descartes, Institut Imagine, Paris, France. 'Service de Maladies Héréditaires du Métabolisme, Centre de Biologie et Pathologie Est, Bron, France. ${ }^{7}$ Centre de Référence des Maladies Héréditaires du Métabolisme, Service de Biochimie, Hôpital Robert-Debré, APHP, Paris, France.

Received: 13 May 2013 Accepted: 10 September 2013

Published: 23 September 2013

\section{References}

1. Deodato F, Boenzi S, Santorelli FM, Dionisi-Vici C: Methylmalonic and propionic aciduria. Am J Med Genet C: Semin Med Genet 2006, 142C:104-112.

2. Grünert SC, Wendel U, Lindner M, Leichsenring M, Schwab KO, Vockley J, Lehnert W, Ensenauer R: Clinical and neurocognitive outcome in symptomatic isovaleric acidemia. Orphanet J Rare Dis 2012, 7:9.

3. Cosson MA, Benoist JF, Touati G, Déchaux M, Royer N, Grandin L, Jais JP, Boddaert N, Barbier V, Desguerre I, Campeau PM, Rabier D, Valayannopoulos $\checkmark$, Niaudet $P$, de Lonlay $P$ : Long-term outcome in methylmalonic aciduria: a series of 30 French patients. Mol Genet Metab 2009, 97:172-178.

4. Touati G, Valayannopoulos V, Mention K, de Lonlay P, Jouvet P, Depondt E, Assoun M, Souberbielle JC, Rabier D, Ogier de Baulny H, Saudubray J-M: Methylmalonic and propionic acidurias: management without or with a few supplements of specific amino acid mixture. J Inherit Metab Dis 2006, 29:288-298.

5. Grünert SC, Müllerleile S, De Silva L, Barth M, Walter M, Walter K, Meissner T, Lindner M, Ensenauer R, Santer R, Bodamer OA, Baumgartner MR, Brunner-Krainz M, Karall D, Haase C, Knerr I, Marquardt T, Hennermann JB, Steinfeld R, Beblo S, Koch H-G, Konstantopoulou V, Scholl-Bürgi S, van Teeffelen-Heithoff A, Suormala T, Sperl W, Kraus JP, Superti-Furga A, Schwab KO, Sass JO: Propionic acidemia: clinical course and outcome in 55 pediatric and adolescent patients. Orphanet J Rare Dis 2013, 8:6.

6. Dercksen M, Duran M, ljlst L, Mienie LJ, Reinecke CJ, Ruiter JPN, Waterham $H R$, Wanders RJA: Clinical variability of isovaleric acidemia in a genetically homogeneous population. J Inherit Metab Dis 2012, 35:1021-1029.

7. De Baulny HO, Benoist JF, Rigal O, Touati G, Rabier D, Saudubray JM: Methylmalonic and propionic acidaemias: management and outcome. J Inherit Metab Dis 2005, 28:415-423. 
8. Dionisi-Vici C, Deodato F, Röschinger W, Rhead W, Wilcken B: "Classical" organic acidurias, propionic aciduria, methylmalonic aciduria and isovaleric aciduria: long-term outcome and effects of expanded newborn screening using tandem mass spectrometry. J Inherit Metab Dis 2006, 29:383-389.

9. Hörster F, Garbade SF, Zwickler T, Aydin HI, Bodamer OA, Burlina AB, Das AM, De Klerk JBC, Dionisi-Vici C, Geb S, Gökcay G, Guffon N, Maier EM, Morava E, Walter JH, Schwahn B, Wijburg FA, Lindner M, Grünewald S, Baumgartner MR, Kölker S: Prediction of outcome in isolated methylmalonic acidurias: combined use of clinical and biochemical parameters. J Inherit Metab Dis 2009, 32:630-639.

10. Brassier A, Boyer O, Valayannopoulos V, Ottolenghi C, Krug P, Cosson MA, Touati G, Arnoux JB, Barbier V, Bahi-Buisson N, Desguerre I, Charbit M, Benoist JF, Dupic L, Aigrain Y, Blanc T, Salomon R, Rabier D, Guest G, de Lonlay $P$, Niaudet P: Renal transplantation in 4 patients with methylmalonic aciduria: A cell therapy for metabolic disease. Mol Genet Metab 2013, 110:106-110.

11. Desviat LR, Pérez B, Pérez-Cerdá C, Rodríguez-Pombo P, Clavero S, Ugarte M: Propionic acidemia: mutation update and functional and structural effects of the variant alleles. Mol Genet Metab 2004, 83:28-37.

12. Pérez B, Desviat $L R$, Rodríguez-Pombo P, Clavero $S$, Navarrete R, Perez-Cerdá C, Ugarte M: Propionic acidemia: identification of twenty-four novel mutations in Europe and North America. Mol Genet Metab 2003, 78:59-67.

13. Martínez MA, Rincón A, Desviat LR, Merinero B, Ugarte M, Pérez B: Genetic analysis of three genes causing isolated methylmalonic acidemia: identification of 21 novel allelic variants. Mol Genet Metab 2005, 84:317-325

14. Vatanavicharn $\mathrm{N}$, Champattanachai V, Liammongkolkul S, Sawangareetrakul P, Keeratichamroen S, Ketudat Cairns JR, Srisomsap C, Sathienkijkanchai A, Shotelersuk V, Kamolsilp M, Wattanasirichaigoon D, Svasti J, Wasant P: Clinical and molecular findings in Thai patients with isolated methylmalonic acidemia. Mol Genet Metab 2012, 106:424-429.

15. Ribeiro CAJ, Balestro F, Grando V, Wajner M: Isovaleric acid reduces Na+, $\mathrm{K}+-$ ATPase activity in synaptic membranes from cerebral cortex of young rats. Cell Mol Neurobiol 2007, 27:529-540.

16. Koekemoer G, Dercksen M, Allison J, Santana L, Reinecke CJ: Concurrent class analysis identifies discriminatory variables from metabolomics data on isovaleric acidemia. Metabolomics 2011, 8:17-28.

17. Schreiber J, Chapman KA, Summar ML, Ah Mew N, Sutton VR, MacLeod E, Stagni K, Ueda K, Franks J, Island E, Matern D, Peña L, Smith B, Urv T, Venditti C, Chakarapani A, Gropman AL: Neurologic considerations in propionic acidemia. Mol Genet Metab 2012, 105:10-15.

18. Okun JG, Hörster F, Farkas LM, Feyh P, Hinz A, Sauer S, Hoffmann GF, Unsicker K, Mayatepek E, Kölker S: Neurodegeneration in methylmalonic aciduria involves inhibition of complex II and the tricarboxylic acid cycle, and synergistically acting excitotoxicity. J Biol Chem 2002, 277:14674-14680.

19. Wajner M, Goodman SI: Disruption of mitochondrial homeostasis in organic acidurias: insights from human and animal studies. J Bioenerg Biomembr 2011, 43:31-38.

20. Kölker S, Schwab M, Hörster F, Sauer S, Hinz A, Wolf NI, Mayatepek E, Hoffmann GF, Smeitink JAM, Okun JG: Methylmalonic acid, a biochemical hallmark of methylmalonic acidurias but no inhibitor of mitochondrial respiratory chain. J Biol Chem 2003, 278:47388-47393.

21. Cosson MA, Touati G, Lacaille F, Valayannnopoulos V, Guyot C, Guest G, Verkarre V, Chrétien D, Rabier D, Munnich A, Benoist JF, de Keyzer Y, Niaudet P, de Lonlay P: Liver hepatoblastoma and multiple OXPHOS deficiency in the follow-up of a patient with methylmalonic aciduria. Mol Genet Metab 2008, 95:107-109.

22. De Keyzer Y, Valayannopoulos V, Benoist J-F, Batteux F, Lacaille F, Hubert L, Chrétien D, Chadefeaux-Vekemans B, Niaudet P, Touati G, Munnich A, de Lonlay P: Multiple OXPHOS deficiency in the liver, kidney, heart, and skeletal muscle of patients with methylmalonic aciduria and propionic aciduria. Pediatr Res 2009, 66:91-95.

doi:10.1186/1750-1172-8-148

Cite this article as: Nizon et al:: Long-term neurological outcome of a cohort of 80 patients with classical organic acidurias. Orphanet Journal of Rare Diseases 2013 8:148.

\section{Submit your next manuscript to BioMed Central and take full advantage of:}

- Convenient online submission

- Thorough peer review

- No space constraints or color figure charges

- Immediate publication on acceptance

- Inclusion in PubMed, CAS, Scopus and Google Scholar

- Research which is freely available for redistribution 